\title{
INTEGERS FREE OF SMALL PRIME FACTORS IN ARITHMETIC PROGRESSIONS*
}

\author{
TI ZUO XUAN
}

\begin{abstract}
For real $x \geq y \geq 2$ and positive integers $a, q$, let $\Phi(x, y ; a, q)$ denote the number of positive integers $\leq x$, free of prime factors $\leq y$ and satisfying $n \equiv a \quad(\bmod q)$. By the fundamental lemma of sieve, it follows that for $(a, q)=1, \Phi(x, y ; a, q)=\varphi(q)^{-1}, \Phi(x, y)\left\{1+O\left(\exp \left(-u\left(\log u-\log _{2} 3 u-2\right)\right)\right)+\right.$ $O(\exp (-\sqrt{\log x} / 2))\}(u=\log x / \log y)$ holds uniformly in a wider ranges of $x$, $y$ and $q$.

Let $\chi$ be any character to the modulus $q$, and $L(s, \chi)$ be the corresponding $L$-function. Let $\tilde{\chi}$ be a ('exceptional') real character to the modulus $q$ for which $L(s, \tilde{\chi})$ have a ('exceptional') real zero $\tilde{\beta}$ satisfying $\tilde{\beta}>1-c_{0} / \log q$. In the paper, we prove that in a slightly short range of $q$ the above first error term can be replaced by $\tilde{\chi}(a) \varphi(q)^{-1} \cdot x^{\tilde{\beta}} \rho^{\prime}(u)(\tilde{\beta} \log y)^{-1}\left(1+O\left((\log y)^{-1 / 2}\right)\right)$, where $\rho(u)$ is Dickman function, and $\rho^{\prime}(u)=d \rho(u) / d u$.

The result is an analogue of the prime number theorem for arithmetic progressions. From the result can deduce that the above first error term can be omitted, if suppose that $1<q<(\log q)^{A}$.
\end{abstract}

\section{$\S 1$. Introduction}

The distribution for integers without large prime factors have been extensively investigated, and have found applications in various problems in number theory (for instance, to finding large gaps between primes, to analysis of algorithms for factoring and primality testing and to Waring's problem).

The dual problem is of studying the distribution of integers free of small prime factors (so-called sifted integers).

To state the results on this problems, we first introduce some notations.

Let $p(n)$ be the smallest prime factor of $n>1$, and $p(1)=\infty$. For real $x \geq y \geq 2$ let $S(x, y)$ denote the set of positive integers $n \leq x$ for which $p(n)>y$, and let $\Phi(x, y)$ denote the cardinality of $S(x, y)$. Also, let $u=\log x / \log y$.

Received April 3, 1998.

${ }^{*}$ Project supported by the National Natural Science Foundation of P. R of China (No. 19571011). 
$\Phi(x, y)$ is an important function of analytic number theory. Various estimates for $\Phi(x, y)$ have been given by several authors (see, [1], [4], [8], [9] [19], [24]), and has a variety of applications (see, [7], [8], [11], [14]).

Thus, it was shown in [4] that for any fixed $u>1$

$$
\Phi(x, y) \sim \frac{x}{\log y} \omega(u) \quad(x \rightarrow \infty)
$$

where the function $\omega(u)$ be defined by

$$
\begin{aligned}
\omega(u) & =1 / u, & & (1 \leq u \leq 2) \\
(u \omega(u))^{\prime} & =\omega(u-1), & & (u \geq 2)
\end{aligned}
$$

where for $u=2$ the right-hand derivative has to be taken.

Recently, Hildebrand [10] derived an asymptotic estimate for $\omega(u)$, where he proved that for $u \geq 2$

$$
\omega(u)-e^{-\gamma}=2 \operatorname{Re}\left\{\frac{-1}{\psi(u)} \Phi(u)\right\}+O\left(\frac{1}{u \lambda(u)}|\Phi(u)|\right),
$$

where

$(1.2) \Phi(u)=\frac{1}{\sqrt{2 \pi u(1-1 / \psi(u))}} \exp \left\{-\gamma-u \psi(u)-\int_{0}^{\psi(u)} \frac{e^{v}-1}{v} d v\right\}$

and where $\psi(u)=\lambda(u)+i \mu(u)$ is the unique solution of $e^{\psi(u)}-1=-u \psi(u)$ in the range $\lambda(u) \geq 2,0<\mu(u) \leq 4 \pi / 3$. Moreover, we have

$$
|\psi(u)| \ll \lambda(u) \ll \log u
$$

Tenenbaum [19, Theorem III.6.7] obtained an estimate for $\Phi(x, y)$ in a wide range. Very recently [24], we extended the range of asymptotic estimate for $\Phi(x, y)$. Then we deduce from the result that the estimate

$$
\begin{aligned}
(1.4) \Phi(x, y) & =x \prod_{p \leq y}\left(1-\frac{1}{p}\right) \\
+ & \frac{x}{\log y}\left(\left(\omega(u)-e^{-\gamma}\right)-\frac{\omega^{\prime}(u-0)}{\log y}+\cdots+\frac{(-1)^{k} \omega^{(k)}(u-0)}{(\log y)^{k}}\right) \\
& +O_{k}\left(\frac{x|\Phi(u)|(\log u)^{k}}{(\log y)^{k+2}}\right) .
\end{aligned}
$$


holds uniformly in a wide range, where $k \geq 0$ is fixed

By (2.7) of [10], we have

$$
|\Phi(u)| / \rho(u)=\exp \left\{-\frac{\pi^{2}}{2} \cdot \frac{u}{\log ^{2} u}(1+o(1))\right\},
$$

where $\rho(u)$ (the so-called "Dickman function") is defined as the continuous solution of the system

$$
\begin{aligned}
\rho(u) & =1 & (0 \leq u \leq 1) \\
-u \rho^{\prime}(u) & =\rho(u-1) & (u>1) .
\end{aligned}
$$

An approximation to $\rho(u)$ in terms of elementary functions $[10,(1.8)]$ is

$$
\begin{aligned}
& \rho(u)= \\
& \exp \left\{-u\left(L+L_{2}-1+\frac{L_{2}}{L}-\frac{1}{L}-\frac{L_{2}^{2}}{2 L^{2}}+\frac{L_{2}}{L^{2}}-\frac{2}{L^{2}}+O\left(\frac{L_{2}^{3}}{L^{3}}\right)\right)\right\},
\end{aligned}
$$

with $L=\log u, L_{2}=\log _{2} u(=\log \log u)$.

A natural problem is to investigate the distribution of the integers free of small prime factors in arithmetic progressions.

In analogy as the function $\Phi(x, y)$, we define

$$
\Phi(x, y ; a, q)=\sum_{\substack{n \in S(x, y) \\ n \equiv a(\bmod q)}} 1, \quad \Phi_{q}(x, y)=\sum_{\substack{n \in S(x, y) \\(n, q)=1}} 1 .
$$

Buchstab [4] considered the function $\Phi(x, y ; a, q)$, and obtained the same result as the case $q=1$ described above.

By the fundamental lemma in the form given in $[9$, Ch.2, Th.2.5], it follows that for $(a, q)=1$

$$
\begin{aligned}
& \Phi(x, y ; a, q)= \\
& \quad \frac{1}{\varphi(q)} \Phi(x, y)\left\{1+O\left(e^{-u\left(\log u-\log _{2} 3 u-2\right)}\right)+O\left(e^{-\frac{1}{2} \sqrt{\log x}}\right)\right\}
\end{aligned}
$$

holds uniformly in the ranges

$$
1 \leq q \leq \sqrt{x}, \quad P(q)<y \text { and } 3 / 2 \leq y \leq x / q,
$$


where $P(n)$ denotes the largest prime factor of $n>1$. The current sieve estimates (see, for example, Iwaniec [13]) give that for $y \geq \exp \left\{(\log x)^{50 / 51}\right\}$ the error terms in (1.7) can be replaced by the bound

$$
e^{-u\left(\log u+\log _{2} 3 u-1+O\left(\log _{2} 3 u / \log u\right)\right)}+\rho(u) \log y .
$$

In [22], Wolke showed that for any $A>0$ the following holds

$$
\sum_{q \leq Q} \max _{(a, q)=1} \max _{z \leq x}\left|\Phi(z, y ; a, q)-\frac{\Phi_{q}(z, y)}{\varphi(q)}\right| \ll x(\log x)^{-A}
$$

with $Q=x^{1 / 2}(\log x)^{-B}$ and $B=B(A)>0$.

In this paper, we will give further estimates for $\Phi(x, y ; a, q)$.

Let $\chi$ be any character to the modulus $q$, and $L(s, \chi)$ be the corresponding $L$-function. Let $\tilde{\chi}$ be a real character to the modulus $q$ for which $L(s, \tilde{\chi})$ have a real zero $\tilde{\beta}$ satisfying $\tilde{\beta}>1-c_{0} / \log q$, where $c_{0}$ is a suitable positive constant. Also let $\chi_{0}$ be the principal to the character modulus $q$.

Let

$$
L_{\varepsilon}(y)=\exp \left\{(\log y)^{3 / 5-\varepsilon}\right\}
$$

where $\varepsilon$ is any fixed positive number.

TheOREM 1. Fix $\varepsilon>0$. Let $x, y$ satisfy

$$
x \geq x_{0}(\varepsilon), \quad \exp \left\{(\log x)^{2 / 5+\varepsilon}\right\} \leq y \leq \sqrt{x}
$$

and

$$
1<q \leq \exp \left\{c_{1} \log y / \log _{2} x\right\}
$$

where $c_{1}$ is a sufficiently small positive constant, and $\chi$ be a nonprincipal character modulus $q$.

(i) If $\chi \neq \tilde{\chi}$, then we have uniformly

$$
\sum_{n \in S(x, y)} \chi(n) \ll x \exp \left\{-c_{2} \log x / \log q\right\}+x / L_{\varepsilon}(x),
$$

where $c_{2}$ is a suitable positive constant, and $L_{\varepsilon}(x)=\exp \left\{(\log x)^{3 / 5-\varepsilon}\right\}$ is defined as in (1.9).

(ii) If $\chi=\tilde{\chi}$, then we have uniformly

$$
\sum_{n \in S(x, y)} \tilde{\chi}(n)=\frac{x^{\tilde{\beta}} \rho^{\prime}(u)}{\tilde{\beta} \log y}\left(1+O\left(\frac{1}{\sqrt{\log y}}\right)\right)+O\left(\frac{x}{L_{\varepsilon}(x)}\right)
$$

where $\rho^{\prime}(u)=d \rho(u) / d u$. 
By [3], we have $\rho^{\prime}(u) \sim-\rho(u) \log u$. Thus, $\rho^{\prime}(u)$ can be estimated by (1.6). In [23], we obtained a sharp asymptotic formula for $\rho^{\prime}(u)$.

Theorem 2. Let $(a, q)=1$. For $x, y$ satisfying $3 / 2 \leq y \leq x / q$, and

$$
1<q \leq \exp \left\{\min \left(c_{3} \sqrt{\log x}, \quad c_{3} \log y / \log _{2} x\right)\right\}, \quad P(q)<y,
$$

where $c_{3}$ is a sufficiently small positive number, we have uniformly

$$
\begin{gathered}
(1.15) \Phi(x, y ; a, q)=\frac{1}{\varphi(q)} \Phi(x, y)+\frac{\tilde{\chi}(a)}{\varphi(q)} \cdot \frac{x^{\tilde{\beta}} \rho^{\prime}(u)}{\tilde{\beta} \log y}\left(1+O\left(\frac{1}{\sqrt{\log y}}\right)\right) \\
+O\left(e^{-\frac{1}{2} \sqrt{\log x}}\right) .
\end{gathered}
$$

We note that though the result (1.7) is stated in the range (1.8), it yields an asymptotic estimate for $\Phi(x, y ; a, q)$ only when $u=\log x / \log y \rightarrow \infty$, as $x \rightarrow \infty$. In Theorem 2, the range of asymptotic estimate for $\Phi(x, y ; a, q)$ is $3 / 2 \leq y \leq x / q$ and (1.14), which is necessary to estimate the sum of form $\sum_{n \in S(x, y), n \equiv a \bmod q} f(n)$, where $f(n)$ is an arithmetic function.

Corollary 1. Let $A>0$ be fixed, $(a, q)=1$. The estimate

$$
\Phi(x, y ; a, q)=\frac{1}{\varphi(q)} \Phi(x, y)\left(1+O\left(e^{-\frac{1}{2} \sqrt{\log x}}\right)\right),
$$

holds uniformly in the ranges $3 / 2 \leq y \leq x / q$, and

$$
1<q \leq(\log x)^{A}, \quad P(q)<y .
$$

We note that Corollary 1 remove the first error term of (1.7).

Theorem 2 is an analogue of the prime number theorem for arithmetic progressions, which can be stated as follows (see, for example, [5, p.123,] and $[16, \mathrm{p} .315]$,$) .$

If we suppose that

$$
q \leq \exp \left\{C(\log x)^{1 / 2}\right\}
$$

where $C$ is any positive constant. Then

$$
\pi(x ; a, q)=\frac{l i x}{\varphi(q)}-\frac{\tilde{\chi}(a)}{\varphi(q)} \int_{2}^{x} \frac{v^{\tilde{\beta}-1}}{\log v} d v+O\left(x e^{-C^{\prime} \sqrt{\log x}}\right)
$$


for a positive constant $C^{\prime}$ depending only on $C$, and this holds uniformly with respect to $q$ in the above range. Evidently, when $y=x^{1 / 2}$, (1.15) gives an asymptotic estimate for the number of primes in an arithmetic progression.

From Theorem 2 and a result of [5, p. 124,], we also get

Corollary 2. On the hypotheses of Theorem 2, then, except possibly if $q$ is a multiple of a particular integer $q_{1}$ depending on $x$, we have uniformly

$$
\Phi(x, y ; a, q)=\frac{1}{\varphi(q)} \Phi(x, y)\left\{1+O\left(e^{-\frac{1}{2} \sqrt{\log x}}\right)\right\} .
$$

We note that Corollary 2 remove the first error term of (1.7), if suppose that $q$ is not an 'exceptional' modulus.

By [5, p. 124], the lower bound for the exceptional modulus $q_{1}$ is

$$
q_{1} \gg \log x /\left(\log _{2} x\right)^{4} \text {. }
$$

\section{$\S 2$. Preliminary lemmas}

Let $q>1$ be integer, $s=\sigma+i t$, put

$$
M(q, t)=\max \left\{\log q, \log ^{2 / 3+\varepsilon}(|t|+3)\right\} .
$$

LEMMA 1. There exists a constant $c_{0}>0$, such that

(i) in the region $\sigma \geq 1-c_{0} / M(q, t)$, there is no zero of any $L(s, \chi)$ with character $\chi(\bmod q)$ except, possibly, one simple real zero of a function $L(s, \tilde{\chi})$ belonging to an exceptional real character $\tilde{\chi}(\bmod q)$.

(ii) in the region $\sigma \geq 1-c_{0} / M(q, t)$, for all nonprincipal character $\chi(\bmod q)$,

$$
L(s, \chi)=O(\log (q(|t|+2))) .
$$

(iii) in the region $\sigma \geq 1-c_{0} / 2 M(q, t),|t| \geq 1$, for all nonprincipal character $\chi(\bmod q)$,

$$
\log L(s, \chi)=O(\log (q(|t|+2))) .
$$

Proof. (i) See [16, Th.17. 4. 2]

(ii) It can be deduced from the estimate (24.2.8) of [16].

(iii) To prove the result, it suffices to show that

$$
\frac{L^{\prime}(\sigma+i t, \chi)}{L(\sigma+i t, \chi)}=\frac{\tilde{E}}{s-\tilde{\beta}}+O(\log (q(|t|+2))),
$$


where $\tilde{\beta}$ is an exceptional real zero of $L(s, \tilde{\chi})$, and $\tilde{E}=1$, if there exists an exceptional character $\tilde{\chi}(\bmod q)$ and $\chi=\tilde{\chi}$, and $\tilde{E}=0$, otherwise,

The proof of the last estimate is almost the same as that of $[17$, Ch.IV, Th.7.1].

Let

$$
L(s, \chi ; y)=\prod_{p \leq y}\left(1-\chi(p) p^{-s}\right)^{-1} .
$$

To prove Theorem 1 , we need an estimate for $L(s, \chi ; y)$. Fouvry and Tenenbaum [6, Lemma 6.3] have given a such estimate, but it would not be sufficient for our purposes (cf. $\S 4$, proof of Theorem 1(ii)). The following lemma gives a slightly different estimate for $L(s, \chi ; y)$.

LEMMA 2. Let $s=\sigma+i t, \sigma, t$ satisfying

$$
\sigma \geq 1-c_{0} / 4 M(q, T), \quad|t| \leq \exp \left\{(\log y)^{3 / 2-\varepsilon}\right\}
$$

where $T=2|t|$, if $|t|>y^{4}$, and $T=2 y^{4}$, if $|t| \leq y^{4}$, and let $q$ satisfying

$$
1 \leq q \leq \exp \left\{c_{6} \log y / \log _{2} y\right\}
$$

where $c_{6}$ is a sufficiently small positive constant. Then we have uniformly for $\chi \neq \chi_{0}(\bmod q)$

$$
L(s, \chi ; y)=e^{-\tilde{E} \tilde{\gamma}(y, s)} E(s, \chi)(1+O(R(y, t, q)))
$$

where $\tilde{E}$ is defined as in the proof of Lemma 1, and where

$$
R(y, t, q)=\left(e^{-c_{5} \log y / M(q, t)}+e^{-c_{5} \log y / M(q, y)}\right) \log ^{2}(q(|t|+y))
$$

here $c_{5}$ is a suitable positive constant, and

$$
\tilde{\gamma}(y, s)=\int_{-\infty}^{\tilde{\beta}-\sigma} \frac{y^{u-i t}}{u-i t} d u \quad(\text { if } \quad t \neq 0) .
$$

Proof. Our method of proof for the lemma has its roots in Vinogradov's approach to the fundamental lemma of [21]. (Also see [24].)

To prove the lemma, we use two different ways to compute the following integral:

$$
J=\frac{1}{2 \pi i} \int_{1-i T}^{1+i T} \frac{y^{w}}{w} \log L(s+w, \chi) d w
$$


where $s=\sigma+i t, t \neq 0, \sigma \geq 1-c_{0} / 4 M(q, T)$.

One side, applying Perron's formula we have

$$
\begin{aligned}
J & =-\sum_{p} \frac{1}{2 \pi i} \int_{1-i T}^{1+i T} \frac{y^{w}}{w} \log \left(1-\frac{\chi(p)}{p^{s+w}}\right) d w \\
& =\log L(s, \chi ; y)+O\left(y^{-1 / 3}\right),
\end{aligned}
$$

where the sum is taken over all primes.

The other side, by residue theorem we have for $\chi \neq \chi_{0}, \tilde{\chi}(\bmod q)$

(2.8) $J=\log L(s, \chi)$

$$
+\frac{1}{2 \pi i}\left(\int_{1-i T}^{-\Delta-i T}+\int_{-\Delta-i T}^{-\Delta+i T}+\int_{-\Delta+i T}^{1+i T}\right) \frac{y^{w}}{w} \log L(s+w, \chi) d w,
$$

where $\Delta=c_{0} / 4 M(q, T)$.

By Lemma 1 and the definition of $T$, the integral along Rew $=-\Delta$ is

$$
\ll e^{-c_{5} \log y / M(q, T)} \log ^{2}(q T) \ll R(y, t, q),
$$

and the integrals along the horizontal sides are $O\left(y^{-2}\right)$.

Hence, for $\chi \neq \chi_{0}, \tilde{\chi}(\bmod q)$, we get

$$
J=\log L(s, \chi)+O(R(y, t, q)) .
$$

If there exists $\tilde{\chi}(\bmod q)$ and $t \neq 0$, we have

(2.11) $J=\log L(s, \tilde{\chi})$

$$
\begin{gathered}
+\frac{1}{2 \pi i}\left(\int_{1-i T}^{-\Delta-i T}+\int_{-\Delta-i T}^{-\Delta+i T}+\int_{-\Delta+i T}^{1+i T}\right) \frac{y^{w}}{w} \log L((s+w, \tilde{\chi}) d w \\
+\frac{1}{2 \pi i} \int_{\Gamma} \frac{y^{w}}{w} \log L(s+w, \tilde{\chi}) d w
\end{gathered}
$$

where $\Gamma$ is a loop starting and finishing at $w=-\Delta-i t$, and encircling the point $w=\tilde{\beta}-s$ in the positive direction.

To estimate $J$ in the case, by the above argument, it only remains to estimate the fourth integral on the right-hand side of (2.12), namely, that along $\Gamma$. We note that the function $L(s+w, \tilde{\chi})$ has a zero $w=\tilde{\beta}-s$, hence $\log \frac{L(s+w, \tilde{\chi})}{w+s-\tilde{\beta}}$ is regular at the point $w=\tilde{\beta}-s$. From this we have

(2.12) $\frac{1}{2 \pi i} \int_{\Gamma} \frac{y^{w}}{w} \log L(s+w, \tilde{\chi}) d w=\frac{-1}{2 \pi i} \int_{\Gamma} \frac{y^{w}}{w} \log \frac{1}{w+s-\tilde{\beta}} d w$. 
Using the argument of [20, pp. 64-65], the integral on right-hand side of $(2.12)$ is equal to

$$
-\int_{-\infty}^{\tilde{\beta}-\sigma} \frac{y^{u-i t}}{u-i t} d u+O(R(y, t, q)) .
$$

So, for $\chi=\tilde{\chi}$, we get

$$
J=\log L(s, \tilde{\chi})-\tilde{\gamma}(y, s)+O(R(y, t, q)) .
$$

The desired estimate (2.3) follows from (2.7), (2.10), (2.13), (2.1) and (2.2), if $c_{6}$ in (2.2) has been chosen sufficiently small. (We note that from this we can deduce $R(y, t, q) \ll 1$, hence $\exp \{O(R(y, t, q))\}=1+O(R(y, t, q))$. )

Remark. To show Theorem 1 , the estimates for $\mathrm{E}(s, \chi ; y)$ are needed. We will apply $(2.3)$, to obtain the estimate for $L(s, \chi) / L(s, \chi ; y)$ in the case $\sigma=1-\delta=1-c_{0} /(4 M(q, T))$, and $|t| \leq L_{\varepsilon}(x)=\exp \left\{(\log x)^{3 / 5+\varepsilon}\right\}$, (see $\S 3,(3.2))$ and $\sigma=\tilde{\beta}-\delta$ and $|t| \leq L_{\varepsilon}(x)$. (See $\S 4$, (4.12) and (4.14).) In the range, the estimate of Fouvry and Tenenbaum [6, Lemma 6.3] would not be sufficient. Moreover, Saias [18] gives an estimate for a related quantity $\zeta(s, y)=\prod_{p \leq y}\left(1-p^{-s}\right)^{-1}$, but the range only to $|t| \ll L_{\varepsilon}(y)$; also Hildebrand and Tenenbaum [12] gives an estimate for the same quantity, but only when $\sigma=$ ' $\alpha$ ' - a saddle point for $\zeta(s, y) x^{s}$, those corresponding estimate would not be sufficient for our purposes.

Thus it can be seen that the lemma of Vinogradov [21] provided a useful tool in this topic, though the paper [21] was criticized. For instance, Norton [15] pointed out that the results stated as case 1) and 2) of Theorem 1 in [21] are incorrect. (In the proof of the lemma in [21] there was some defect, so we [23] have given a summary of the proof once more.)

Let

$$
\gamma(y, s)=\int_{-\infty}^{1-\sigma} \frac{y^{u-i t}}{u-i t} d u \quad(t \neq 0) .
$$

From (2.5) we have

$$
\tilde{\gamma}(y, s)=\gamma(y, 1+\sigma-\tilde{\beta}+i t) .
$$

LEMMA 3. On the hypotheses of Lemma 2 we have

$$
\begin{aligned}
L(s, \tilde{\chi} ; y)=\exp \{-\gamma & \left.-\int_{0}^{(\tilde{\beta}-s) \log y} \frac{e^{v}-1}{v} d v\right\} \\
& \times \frac{L(s, \tilde{\chi})}{(s-\tilde{\beta}) \log y}(1+O(R(y, t, q))) .
\end{aligned}
$$


Proof. By Lemma 2, to prove the lemma, it suffices to show that

$$
\gamma(y, s)=\pi i+\gamma+I((1-s) \log y)+\log ((1-s) \log y),
$$

where

$$
I(s)=\int_{0}^{s}\left(e^{v}-1\right) v^{-1} d v
$$

We can write

$$
\gamma(y, s)=\int_{-\infty-i t}^{1-s} y^{w} w^{-1} d w \quad(t \neq 0) .
$$

By Cauchy's theorem, we have

$$
\gamma(y, s)+\int_{\Gamma_{1}+\Gamma_{2}+\Gamma_{3}} y^{w} w^{-1} d w=2 \pi i
$$

where $\Gamma_{i}(1 \leq i \leq 3)$ are defined as follows:

(i) $\Gamma_{1}$ is a line segment from $1-s$ to $1-\sigma$;

(ii) $\Gamma_{2}$ is a semi circle starting at $w=1-\sigma$ and finishing at $w=-(1-\sigma)$, and encircling the origin $w=0$ in the positive direction;

(iii) $\Gamma_{3}$ is a line segment from $-(1-\sigma)$ to $-\infty$.

Clearly,

$$
\int_{\Gamma_{2}} w^{-1} d w=\pi i
$$

From (2.17) and (2.18) we get

$\gamma(y, s)=\pi i+\int_{1-\sigma}^{1-s} \frac{y^{w}}{w} d w+\int_{-(1-\sigma)}^{1-\sigma} \frac{y^{v}-1}{v} d v-\int_{-(1-\sigma)}^{-\infty} \frac{y^{v}}{v} d v$

It is well known that $\Gamma^{\prime}(1)=-\gamma$, where $\Gamma(s)$ denotes the gammafunction. It follows that

$$
-\gamma=\int_{0}^{1}\left(e^{-v}-1\right) v^{-1} d v+\int_{1}^{+\infty} e^{-v} v^{-1} d v
$$

Thus, the desired estimate (2.16) follows from (2.19) and (2.20).

This completes the proof of Lemma 3. 
By Lemma 3 and the definition of $\tilde{\gamma}(y, s)$, we have

$$
\tilde{\gamma}(y, s)=\gamma+I((\tilde{\beta}-s) \log y)+\log ((s-\tilde{\beta}) \log y) .
$$

Let $\xi(u)$ denote the positive solution of the equation $e^{\xi}=u \xi+1(u>1)$. Then we have $\xi(u)=\log u+\log _{2} u+O\left(\log _{2} u / \log u\right)$.

To further estimate $\tilde{\gamma}(y, s)$, for $s=B+i t=\tilde{\beta}-\xi(u) / \log y+i t$, it can be written as

$$
\tilde{\gamma}(y, B+i t)=\gamma+I(\xi(u))+w(u,-i t \log y)+\log (-\xi(u)),
$$

where

$$
w(u, z)=\int_{0}^{z} e^{\xi(u)+w}(\xi(u)+w)^{-1} d w .
$$

Let $a(u, t)=\operatorname{Re} w(u,-i t)$. The following lemmas give the estimates for $a(u, t)$, which are proved in [23].

Lemma 4. For $u \geq 2, t \geq 1$ we have uniformly

$$
e^{a(u, t)} \ll e^{-u /\left(9 \log ^{2} u\right)} .
$$

LEMMA 5. For $u \geq 2,0 \leq t \leq 1$ we have uniformly

$$
e^{a(u, t)} \ll e^{-c_{7} u t^{2}}
$$

where $c_{7}$ is a sufficiently small positive constant.

\section{§. Proof of Theorem 1 (i)}

Perron's formula gives

$$
\sum_{n \in S(x, y)} \chi(n)=\frac{1}{2 \pi i} \int_{\sigma_{1}-i T}^{\sigma_{1}+i T} \frac{L(s, \chi)}{L(s, \chi ; y)} \frac{x^{s}}{s} d s+O\left(\frac{x \log x}{T}\right)
$$

where $\sigma_{1}=1+(1 / \log x)$ and $T=L_{\varepsilon}^{2}(x)=\exp \left\{2(\log x)^{3 / 5-\varepsilon}\right\}$. Suppose $\chi \neq \tilde{\chi}(\bmod q)$. By Cauchy's theorem we have

$$
\frac{1}{2 \pi i} \int_{\sigma_{1}-i T}^{\sigma_{1}+i T} \frac{L(s, \chi)}{L(s, \chi ; y)} \frac{x^{s}}{s} d s=\frac{1}{2 \pi i}\left\{\int_{1-\delta-i T}^{1-\delta+i T}+\int_{1-\delta+i T}^{\sigma_{1}+i T}+\int_{\sigma_{1}-i T}^{1-\delta-i T}\right\}
$$

where $\delta=c_{0} /(4 M(q, T))$. By Lemma 2 we have, for $\chi \neq \tilde{\chi}$ and $x, y, q$ satisfying (1.10) and (1.11), respectively,

$$
L(s, \chi) / L(s, \chi ; y) \ll 1 .
$$


Hence, the integral along $\operatorname{Re} s=1-\delta$ is

$$
\ll \int_{1-\delta-i T}^{1-\delta+i T}\left|\frac{x^{s}}{s}\right||d s| \ll x \exp \left\{-\frac{c_{0} \log x}{4 M(q, T)}\right\} \log (q T) .
$$

By the definitions of $M(q, T)$ and $T$, we have

$$
M(q, T)=\max \left\{\log q,(\log x)^{2 / 5+\varepsilon}\right\} .
$$

So, the right-hand side of (3.2) is bounded by

$$
\ll x \exp \left\{-c_{2} \log x / \log q\right\}+x / L_{\varepsilon}(x) .
$$

Moreover, the integrals along the horizontal sides are

$$
\ll \int_{1-\delta}^{\sigma_{1}} T^{-1} x^{\sigma} d \sigma \ll x / L_{\varepsilon}(x) .
$$

Combining these estimates we obtain (1.12). This completes the proof of part (i) of Theorem 1.

$\S 4$. Proof of Theorem 1 (ii): the case $u>\log _{2}^{2} y$

To prove the theorem, we need the following lemma.

LEMMA 6. For $u \geq 2$, we have uniformly

$$
\rho^{\prime}(u)=e^{\gamma-u \xi(u)+I(\xi(u))}(-\xi(u) \log y) J(u)+O\left(E_{a}\right),
$$

where

$$
J(u)=\frac{1}{2 \pi} \int_{-1}^{1} e^{w(u,-i \bar{t})+i \bar{t} u} d t
$$

$(\bar{t}=t \log y)$ and

$$
E_{a}=e^{-u \xi(u)+I(\xi(u))-c_{8} u / \log ^{2} u}
$$

where $c_{8}$ is a suitable positive constant.

Proof. By (1.9) of [2] we have

$$
\rho(u)=\frac{1}{2 \pi i} \int_{-i \infty}^{i \infty} e^{\gamma-u s+I(s)} d s \quad(u \geq 1) .
$$


From this, (3.3) and (3.4) of [2] we obtain for $T \geq 1, u \geq 1$

$$
\rho(u)=\frac{1}{2 \pi i} \int_{-i T}^{i T} e^{\gamma-u s+I(s)} d s+O\left(T^{-1}\right) .
$$

Form (4.2) and the definition of $\rho(u)$ we get

$$
\rho^{\prime}(u)=\frac{-1}{2 \pi i u} \int_{-i T}^{i T} e^{\gamma-(u-1) s+I(s)} d s+O\left(T^{-1}\right) .
$$

Since

$$
\operatorname{Re} I(i T)=\int_{0}^{T} \frac{\cos t-1}{t} d t=-\log T+O(1)
$$

and

$$
I(\sigma+i T)-I(i T) \ll T^{-1} \int_{0}^{\xi(u)} e^{\sigma} d \sigma \ll 1,
$$

if $T \geq e^{\xi(u)}$ and $0 \leq \sigma \leq \xi(u)$, so we have

$$
\rho^{\prime}(u)=-u^{-1} e^{\gamma-(u-1) \xi(u)+I(\xi(u))} J_{1}(u)+O(1 / T),
$$

where

$$
J_{1}(u)=\frac{1}{2 \pi} \int_{-T}^{T} e^{-i t(u-1)+I(\xi(u)+i t)-I(\xi(u))} d t .
$$

Obviously

$$
I(\xi(u)+i t)-I(\xi(u))=\int_{0}^{i t} \frac{e^{\xi(u)+w}}{\xi(u)+w} d w+\log \left(\frac{\xi(u)}{\xi(u)+i t}\right) .
$$

So, by the definition of $w(u, z)$, we have

$$
J_{1}(u)=\frac{1}{2 \pi} \int_{-T}^{T} \frac{e^{-i t(u-1)+w(u, i t)}}{1+(i t / \xi(u))} d t .
$$

Choose $T=e^{2 u \xi(u)} \log y$. We split the range into the two parts: $|t| \leq \log y$ and $\log y<|t| \leq T$, the corresponding integral being denoted by $J_{2}$ and $J_{3}$. By Lemma 4 we have

(4.9) $J_{3} \ll \int_{\log y}^{T} \frac{\left|e^{w(u, i t)}\right|}{|1+(i t / \xi(u))|} d t \ll e^{-u /\left(10 \log ^{2} u\right)} \log y \ll e^{-c_{8} u / \log ^{2} u}$, since $u>\log _{2}^{2} y$. 
To estimate $J_{2}$, we note that the integrand can be written as

$$
(-i \xi(u)) e^{-\xi(u)-i t u} d e^{w(u, i t)} / d t .
$$

Partial integration and Lemma 4 give

$$
\text { (4.10) } J_{2}=u \xi(u) e^{-\xi(u)}\left\{\frac{1}{2 \pi} \int_{-\log y}^{\log y} e^{-i t u+w(u, i t)} d t+O\left(e^{-c_{8} u / \log ^{2} u}\right)\right\} \text {. }
$$

Lemma 6 follows from (4.6) and (4.8)-(4.10).

\section{Proof of Theorem 1 (ii): the case $u>\log _{2}^{2} y$}

For $\chi=\tilde{\chi}(\bmod q)$, by $(3.1)$, we have

$$
\sum_{n \in S(x, y)} \tilde{\chi}(n)=\frac{1}{2 \pi i} \int_{\sigma_{1}-i T}^{\sigma_{1}+i T} \frac{L(s, \tilde{\chi})}{L(s, \tilde{\chi} ; y)} \frac{x^{s}}{s} d s+O\left(\frac{x}{L_{\varepsilon}(x)}\right) .
$$

By Cauchy's theorem, the integral on the right-hand side in (4.11) may be replaced by integrals $I_{1}, \cdots, I_{9}$ over paths $\Gamma_{1}, \cdots, \Gamma_{9}$ which are defined as follows:

$\Gamma_{1}$ is a line segment from $\tilde{\beta}-\delta+i T$ to $\sigma_{1}+i T$, with $T=L_{\varepsilon}(x), \quad \delta=$ $c_{0} /(4 M(q, T))$;

$\Gamma_{2}$ is a line segment from $\tilde{\beta}-\delta+i T_{a}$ to $\tilde{\beta}-\delta+i T$; with

$$
T_{a}=\frac{c_{8}}{\log x} \exp \left\{\frac{c_{0} \log y}{4 M(q, T)}\right\}
$$
to $T_{a}$;

$\Gamma_{3}$ is a curve described by $\tilde{\beta}-\frac{\log \left(c_{8}^{-1} t \log x\right)}{\log y}+i t$, as $t$ increases from 1

$\Gamma_{4}$ is a line segment from $B+i$ to $\tilde{\beta}-\log \left(c_{8}^{-1} \log x\right) / \log y+i$, with $B=\tilde{\beta}-\xi(u) / \log y$;

$\Gamma_{5}$ is a line segment from $B-i$, to $B+i$;

$\Gamma_{6}$ is a line segment from $\tilde{\beta}-\log \left(c_{8}^{-1} \log x\right) / \log y-i$ to $B-i$;

$\Gamma_{7}$ is a curve described by $\tilde{\beta}-\frac{\log \left(-c_{8}^{-1} t \log x\right)}{\log y}+i t$, as $t$ increases from $-T_{a}$ to -1 ,

$\Gamma_{8}$ is a line segment from $\tilde{\beta}-\delta-i T$ to $\tilde{\beta}-\delta-i T_{a}$;

$\Gamma_{9}$ is a line segment from $\sigma_{1}-i T$ to $\tilde{\beta}-\delta-i T$.

By Lemma 2 we have, on $\Gamma_{2}$

$$
\begin{aligned}
|L(s, \tilde{\chi}) / L(s, \tilde{\chi} ; y)| & \ll e^{|\tilde{\gamma}(y, s)|} \ll \exp \left\{y^{\tilde{\beta}-\sigma}(|t| \log y)^{-1}\right\} \\
& \ll \exp \left\{y^{\delta}(|t| \log y)^{-1}\right\} \ll e^{u / c_{8}}
\end{aligned}
$$


From this and (3.3) we have

$$
\begin{aligned}
I_{2} & \ll \int_{T_{a}}^{T} x^{\tilde{\beta}} e^{-\delta \log x+u / c_{8}} t^{-1} d t \\
& \ll x^{\tilde{\beta}} \exp \left\{-c_{9}(\log x)^{3 / 5-\varepsilon}\right\} \log T+x^{\tilde{\beta}} \exp \left\{-c_{9} \log x / \log q\right\} \log T .
\end{aligned}
$$

(1.10) and (1.11) give

$$
c_{9} \log x / \log q>c_{9} c_{1} u \log _{2} x>2 u \log u
$$

and

$$
2 u \log u \leq(\log x)^{3 / 5-\varepsilon / 2} .
$$

By the definitions of $\xi(u)$ and $I(s)$ we have $\xi(u)=\log u+O\left(\log _{2} u\right)$ and $I(\xi(u))=O(u)$. From the above estimates and $u>\log _{2}^{2} y$ we deduce that

$$
I_{2} \ll x^{\tilde{\beta}} e^{-u \xi(u)+I(\xi(u))}(\log y)^{-1} \cdot e^{-c_{10} u / \log ^{2} u}(\log y)^{-1}:=E_{1} .
$$

Also, it is easy to estimate $I_{1} \ll E_{1}$.

On $\Gamma_{3}$ we have, by Lemma 2

$$
|L(s, \tilde{\chi}) / L(s, \tilde{\chi} ; y)| \ll e^{|\tilde{\gamma}(y, s)|} \ll \exp \left\{y^{\tilde{\beta}-\sigma}(|t| \log y)^{-1}\right\} \ll e^{u / c_{8}}
$$

From this we further have

$$
I_{3} \ll x^{\tilde{\beta}} \int_{1}^{T_{a}} e^{-u \log \left(c_{8}^{-1} t \log x\right)} e^{u / c_{8}} d t \ll x^{\tilde{\beta}} e^{-(3 u / 4) \log _{2} x} \ll E_{1}
$$

here we have used (1.10).

For $I_{4}$, we readily get

$$
I_{4} \ll \int_{1 / 2}^{B} x^{\sigma} e^{u / c_{8}} d \sigma \ll x^{\tilde{\beta}} e^{-u \xi(u)+u / c_{8}}(\log y)^{-1} \ll E_{1},
$$

since $u>\log _{2}^{2} y$.

For $I_{j}(j=6,7,8,9)$, by the same argument as before we get that they can be bounded by the right-hand side of (4.13). So, we obtain

$$
\sum_{n \in S(x, y)} \tilde{\chi}(n)=I_{5}+O\left(E_{1}\right)+O\left(x / L_{\varepsilon}(x)\right)
$$


where

$$
I_{5}=\frac{1}{2 \pi i} \int_{B-i}^{B+i} \frac{L(s, \tilde{\chi})}{L(s, \tilde{\chi} ; y)} \frac{x^{s}}{s} d s .
$$

To estimate $I_{5}$, we note that by $B=\tilde{\beta}-\xi(u) / \log y$ we have, for $s=B+i t,(\tilde{\beta}-s) \log y=\xi(u)-i t \log y$. So, by Lemma 2 and $(2.22)$, the integral $I_{5}$ can be written as

$$
I_{5}=x^{\tilde{\beta}} Q(u) \cdot \frac{1}{2 \pi} \int_{-1}^{1} \frac{e^{w(u,-i \bar{t})+i \bar{t} u}}{B+i t}(1+O(R(y, t, q))) d t
$$

where $\bar{t}=t \log y, Q(u)=e^{\gamma-u \xi(u)+I(\xi(u))}(-\xi(u))$ and $w(u,-i \bar{t})$ is defined as in $\S 2$.

By Lemmas 4 and 5 we have $\left(e^{w(u,-i \bar{t})+i \bar{t} u}\right) /(B+i t) \ll 1$. From the definition of $R(y, t, q)$ and (1.11), it follows that

$$
R(y, t, q) \ll e^{-c_{2} \log y / \log q}+e^{-(\log y)^{\varepsilon}} \ll(\log y)^{-N} .
$$

Hence, the contribution of the error term $O(R(y, t, q))$ to the integral in $(4.19)$ is

$$
\ll x^{\tilde{\beta}} Q(u)(\log y)^{-N} .
$$

From this, (4.17) and (4.19) we obtain

$$
\sum_{n \in S(x, y)} \tilde{\chi}(n)=x^{\tilde{\beta}} Q(u) \cdot \frac{1}{2 \pi} \int_{-1}^{1} e^{w(u,-i \bar{t})+i \bar{t} u}(B+i t)^{-1} d t+O\left(E_{1}\right)
$$

By Lemma 6 we have, for $u>\log _{2}^{2} y$,

$$
\rho^{\prime}(u)=Q(u) \log y \cdot \frac{1}{2 \pi} \int_{-1}^{1} e^{w(u,-i \bar{t})+i \bar{t} u} d t+O\left(E_{b}\right)
$$

where

$$
E_{b}=e^{-u \xi(u)+I(\xi(u))-c_{11} u / \log ^{2} u}(\log y)^{-1} .
$$

Moreover, it is well known (see [2], (1.6)) that

$$
\rho^{\prime}(u) \sim(-\log u) \rho(u) \asymp u^{-1 / 2} \log u \cdot e^{-u \xi(u)+I(\xi(u))}
$$

Hence, in order to prove the theorem, it suffices to show that

$$
\frac{1}{2 \pi} \int_{-1}^{1} e^{w(u,-i \bar{t})+i \bar{t} u}\left(\frac{1}{B+i t}-\frac{1}{\tilde{\beta}}\right) d t \ll \frac{1}{\sqrt{u} \log y} \frac{\log u}{\log y} .
$$


We have $1 /(B+i t)-1 / \tilde{\beta} \ll \xi(u) / \log y+t$. From this, Lemmas 4 and 5 we deduce that the integral in $(4.24)$ is

$$
\begin{aligned}
& \ll \int_{0}^{1 / \log y} e^{-c_{6} u(t \log y)^{2}}(\xi(u) / \log y+t) d t+\int_{1 / \log y}^{1} e^{-u /\left(9 \log ^{2} u\right)} d t \\
& \ll \frac{1}{\sqrt{u} \log y} \cdot \frac{\log u}{\log y}+e^{-u / 9 \log ^{2} u} \ll \frac{1}{\sqrt{u} \log y} \cdot \frac{\log u}{\log y},
\end{aligned}
$$

since $u>\log _{2}^{2} y$. The desired estimate (4.24) follows.

This completes the proof of Theorem 1(ii) in the range considered.

$\S 5$. Proof of Theorem 1 (ii): the case $2 \leq u \leq \log _{2}^{2} y$

We need the following lemmas.

LEMMA 7. For $s=\sigma+i t, \sigma>0,|t| \geq 1$, we have uniformly

$$
e^{I(\sigma+i t)} \ll(|\sigma+i t|)^{-1} \exp \left\{e^{\sigma}(|t|)^{-1}\right\} .
$$

Proof. We may suppose without loss of generality that $t \geq 1$. We have

$$
I(\sigma+i t)=\int_{0}^{1} \frac{e^{v}-1}{v} d v+\int_{1}^{i} \frac{e^{v}}{v} d v+\int_{i}^{i t} \frac{e^{v}}{v} d v+K_{1}-\log (\sigma+i t),
$$

where

$$
K_{1}=\int_{i t}^{\sigma+i t} e^{v} v^{-1} d v=\int_{0}^{\sigma} e^{v+i t}(v+i t)^{-1} d v
$$

Hence

$$
\left|K_{1}\right| \leq \int_{0}^{\sigma} e^{v}(|t|)^{-1} d v \leq|t|^{-1} e^{\sigma}
$$

The Lemma follows from the above estimates.

LEMMA 8. For $2 \leq u \leq\left(\log _{2} y\right)^{2}$ we have uniformly

$$
\rho^{\prime}(u)=\frac{-1}{2 \pi i} \int_{L} e^{\gamma-u s+I(s)+\log s} d s+O\left(E_{b}\right),
$$

where $E_{b}$ is defined as in (4.22) and contour $L$ will be given in (5.6) below. 
Proof. By Cauchy's theorem the integral on the right-hand side in (4.3) may be replaced by integrals $J_{1}, \cdots, J_{9}$ over paths $L_{1}, \cdots, L_{9}$ which are defined as follows:

$L_{1}$ is a line segment from $2 \log _{2} y+i T$ to $i T$, with $T=e^{2 u \xi(u)} \log ^{2} y$;

$L_{2}$ is a line segment from $2 \log _{2} y+i T_{1}$ to $2 \log _{2} y+i T$, with $T_{1}=\log ^{2} y$;

$L_{3}$ is a curve described by $\log |t|+i t$ as $t$ increases from $T_{2}$ to $T_{1}$, where $T_{2}=\log y$;

$L_{4}$ is the same curve, as $t$ increases from $T_{3}$ to $T_{2}$, where $T_{3}=e^{\xi(u)}$;

$L_{5}$ is a line segment from $\xi(u)-i T_{3}$, to $\xi(u)+i T_{3}$;

$L_{6}$ is a curve described by log $|t|+i t$ as $t$ increases from $-T_{2}$ to $-T_{3}$;

$L_{7}$ is the same curve as $t$ increases from $-T_{1}$ to $-T_{2}$;

$L_{8}$ is a line segment from $2 \log _{2} y-i T$ to $2 \log _{2} y-i T_{1}$;

$L_{9}$ is a line segment from $-i T$ to $2 \log _{2} y-i T$.

By Lemma 7 and (4.3) we get

$$
\begin{aligned}
J_{2} & =\frac{-1}{2 \pi u} \int_{T_{1}}^{T} e^{\gamma-(u-1)\left(2 \log _{2} y+i t\right)+I\left(2 \log _{2} y+i t\right)} d t+O\left(T^{-1}\right) \\
& \ll u^{-1} \int_{T_{1}}^{T} e^{-2(u-1) \log _{2} y} t^{-1} d t \ll u^{-1} e^{-2(u-1) \log _{2} y} u^{2} \\
& \ll u^{-1}(\log y)^{1-u} .
\end{aligned}
$$

If $10<u \leq\left(\log _{2} y\right)^{2}$, then the above bound becomes

$$
\ll u^{-1} e^{-u \xi(u)}(\log y)^{(1-u) / 2} \ll E_{b} .
$$

If $2 \leq u \leq 10$, we easily see that the same estimate holds.

Similarly, we can show that the same is true for the integral $J_{1}$.

We now show that the integral $J_{3}$ is bounded by $E_{b}$. By Lemma 7 we have

$$
J_{3} \ll u^{-1} \int_{T_{2}}^{T_{1}} e^{-(u-1) \log t} t^{-1} d t \ll u^{-1}(\log y)^{1-u},
$$

and the desired bound follows.

Similarly, we can show that the integrals $J_{j}(j=7,8,9)$ is bounded by $E_{b}$.

Thus, we obtain

$$
\rho^{\prime}(u)=\frac{-1}{2 \pi i u} \int_{L} e^{\gamma-(u-1) s+I(s)} d s+O\left(E_{b}\right),
$$


where

$$
L=L_{4}+L_{5}+L_{6}
$$

To estimate the integral in (5.5), we note that

$$
I(s)=I(1)+E(s)-\log s,
$$

where

$$
E(s)=\int_{1}^{s} e^{v} v^{-1} d v .
$$

Hence, the integral in (5.5) equals

$$
\begin{aligned}
& \frac{-1}{2 \pi u} \int_{-T_{2}}^{T_{2}} Q_{1}(u) e^{-i t(u-1)+E\left(\sigma_{2}+i t\right)}\left(\sigma_{2}+i t\right)^{-1} d t \\
& \quad=\frac{-1}{2 \pi u} \int_{-T_{2}}^{T_{2}} Q_{1}(u)\left(-i e^{-\sigma_{2}}\right) e^{-i t u} \frac{d}{d t}\left(e^{E\left(\sigma_{2}+i t\right)}\right) d t
\end{aligned}
$$

where $\sigma_{2}=\log _{2} y$, and

$$
Q_{1}(u)=e^{\gamma-(u-1) \sigma_{2}+I(1)}
$$

By using integration by parts this is

$$
\frac{-1}{2 \pi} \int_{-T_{2}}^{T_{2}} Q_{1}(u) e^{-i t u-\sigma_{2}+E\left(\sigma_{2}+i t\right)} d t+O\left(E_{b}\right)
$$

here we have used the estimate:

$$
e^{E\left(\sigma_{2}+i T_{2}\right)} \ll e^{I\left(\sigma_{2}+i T_{2}\right)}\left|\sigma_{2}+i T_{2}\right| \ll 1,
$$

by Lemma 7 . The desired estimate follows from this and (5.5)-(5.7).

Proof of Theorem 1 (ii): the case $2 \leq u \leq \log { }_{2}^{2} y$.

We note that in the range considered, (4.11) is still valid. The integral on the right-hand side of (4.11) may be replaced by integrals $I_{1}^{\prime}, \cdots, I_{9}^{\prime}$ over paths $\Gamma_{1}^{\prime}, \cdots, \Gamma_{9}^{\prime}$ which are defined as follows:

$\Gamma_{1}^{\prime}$ is a line segment from $\tilde{\beta}-\delta+i T$ to $\sigma_{1}+i T$, with $T=L_{\varepsilon}(x)$ and $\delta=c_{0} /(4 M(q, T))$;

$\Gamma_{2}^{\prime}$ is a line segment from $\tilde{\beta}-\delta+i T_{a}^{\prime}$ to $\tilde{\beta}-\delta+i T$, with $T_{a}^{\prime}=$ $\frac{1}{\log y} \exp \left\{\frac{c_{0} \log y}{4 M(q, T)}\right\}$; 

$T_{a}^{\prime}$

$\Gamma_{3}^{\prime}$ is a curve described by $\tilde{\beta}-\frac{\log (|t| \log y)}{\log y}+i t$, as $t$ increases from 1 to

$\Gamma_{4}^{\prime}$ is the same curve, as $t$ increases $T_{b}$ to 1 , with $T_{b}=e^{\xi(u)}(\log y)^{-1}$;

$\Gamma_{5}^{\prime}$ is a line segment from $B-i T_{b}$ to $B+i T_{b}$, with $B=\tilde{\beta}-\xi(u) / \log y$;

$\Gamma_{6}^{\prime}$ is a curve described by $\tilde{\beta}-\frac{\log (|t| \log y)}{\log y}+i t$, as $t$ increases from -1 to $-T_{b}$;

$\Gamma_{7}^{\prime}$ is the same curve, as $t$ increases $-T_{a}^{\prime}$ to -1 ,

$\Gamma_{8}^{\prime}$ is a line segment from $\tilde{\beta}-\delta-i T$ to $\tilde{\beta}-\delta-i T_{a}^{\prime}$;

$\Gamma_{9}^{\prime}$ is a line segment from $\sigma_{1}-i T$ to $\tilde{\beta}-\delta-i T$.

We note that in the range considered, (4.13),(4.16) is still valid, namely $I_{1}^{\prime}+I_{2}^{\prime}+I_{3}^{\prime} \ll E_{1}$, and similarly $I_{7}^{\prime}+I_{8}^{\prime}+I_{9}^{\prime} \ll E_{1}$. Hence, we have

$$
\sum_{n \in S(x, y)} \tilde{\chi}(n)=I_{4}^{\prime}+I_{5}^{\prime}+I_{6}^{\prime}+O\left(E_{1}\right)
$$

where

$$
I_{j}^{\prime}=\frac{1}{2 \pi i} \int_{\Gamma_{j}^{\prime}} \frac{L(s, \tilde{\chi})}{L(s, \tilde{\chi} ; y)} \frac{x^{s}}{s} d s, \quad(j=4,5,6) .
$$

Moreover, by Lemma 8, we have

$$
\rho^{\prime}(u)=\frac{1}{2 \pi i} \int_{L} e^{\gamma-u \bar{s}+I(\bar{s})}(-\bar{s}) d s+O\left(E_{b}\right)=J_{4}^{\prime}+J_{5}^{\prime}+J_{6}^{\prime}+O\left(E_{b}\right),
$$

where $s=\sigma+i t, \bar{s}=\sigma-i t$ and

$$
J_{j}^{\prime}=\frac{-1}{2 \pi i} \int_{L_{j}} e^{\gamma-u \bar{s}+I(\bar{s})} \bar{s} d s, \quad(j=4,5,6) .
$$

Hence, in order to prove Theorem 1 (ii) in the range considered, it suffices to show that

$$
I_{j}^{\prime}-x^{\tilde{\beta}}(\tilde{\beta} \log y)^{-1} \cdot J_{j}^{\prime} \ll E_{2}, \quad(j=4,5,6),
$$

where

$$
E_{2}=x^{\tilde{\beta}} Q(u)(\sqrt{u} \log y)^{-1} \cdot(\log y)^{-1 / 2},
$$

and here $Q(u)$ is defined as in (4.19).

We first consider the case when $j=4$. By Lemma 3, the integral $I_{4}^{\prime}$ can be written as

$$
I_{4}^{\prime}=x^{\tilde{\beta}} \cdot \frac{1}{2 \pi} \int_{T_{b}}^{1} F(u, \bar{t}) s^{-1}(1+O(R(y, t, q))) d t
$$


where $s=\tilde{\beta}-(\log |\bar{t}|) / \log y+i t$, and

$$
F(u, \bar{t})=e^{\gamma+I(\log |\bar{t}|-i \bar{t})-u \log |\bar{t}|+i \bar{t} u}(-\log |\bar{t}|+i \bar{t}) .
$$

By Lemma 7 we have, for $T_{b} \leq|t| \leq 1$,

$$
|F(u, \bar{t})| \ll e^{-u \log |\bar{t}|} \exp \left\{|\bar{t}|^{-1} e^{\log |\bar{t}|}\right\} \ll(t \log y)^{-u} .
$$

Moreover, the integral $J_{4}^{\prime}$ can be written as

$$
J_{4}^{\prime}=\log y \cdot \frac{1}{2 \pi} \int_{T_{b}}^{1} F(u, \bar{t}) d t .
$$

From $\S 4$ we know that $R(y, t, q) \ll(\log x)^{-N}$, and

$$
(1 / s)-(1 / \tilde{\beta}) \ll(|\log \bar{t}| / \log y)+t .
$$

Thus, from this, (5.14), (5.16) and (5.17) we obtain

$$
\begin{aligned}
& I_{4}^{\prime}-x^{\tilde{\beta}}(\tilde{\beta} \log y)^{-1} J_{4}^{\prime} \\
= & x^{\tilde{\beta}} \cdot \frac{1}{2 \pi} \int_{T_{b}}^{1} F(u, \bar{t})\left\{s^{-1}(1+O(R(y, t, q)))-\tilde{\beta}^{-1}\right\} d t \\
& \ll \frac{x^{\tilde{\beta}}}{(\log y)^{u}} \int_{T_{b}}^{1}\left(\frac{|\log \bar{t}|}{\log y}+t\right) \frac{d t}{t^{u}} \ll \frac{x^{\tilde{\beta}}}{(\log y)^{u}} \int_{T_{b}}^{1} \frac{d t}{t^{u-1}} .
\end{aligned}
$$

When $u \geq 5 / 2$, the last integral is

$$
\ll T_{b}^{-u+2} \ll(\log y)^{u-2} e^{-(u-2) \xi(u)} .
$$

(We recall that $T_{b}=e^{\xi(u)}(\log y)^{-1}$.) Hence, we find that the right-hand side of (5.18) is

$$
\ll x^{\tilde{\beta}} e^{-u \xi(u)+I(\xi(u))-u / 2}(\log y)^{-3 / 2} \ll E_{2} .
$$

If $2 \leq u \leq 5 / 2$, the last estimate remains true, since, for $T_{b} \leq t \leq 1$, we have $t^{-u+1} \leq t^{-3 / 2}$ and $\int_{T_{b}}^{1} t^{-3 / 2} d t \ll \sqrt{\log y}$. Thus, (5.12) follows for $j=4$. Similarly, (5.12) holds for $j=6$.

It remains to prove estimates (5.12) for $j=5$.

It follows from Lemma 2 and (2.22) that

$$
I_{5}^{\prime}=x^{\tilde{\beta}} Q(u) \cdot \frac{1}{2 \pi} \int_{-T_{b}}^{T_{b}} \frac{e^{w(u,-i \bar{t})+i \bar{t} u}}{B+i t}(1+O(R(y, t, q))) d t .
$$


By the relation

$$
I(\xi(u)-i \bar{t})=I(\xi(u))+w(u,-i \bar{t})+\log \left(\frac{\xi(u)}{\xi(u)-i \bar{t}}\right),
$$

we have

$$
J_{5}^{\prime}=(\log y) Q(u) \cdot \frac{1}{2 \pi} \int_{-T_{b}}^{T_{b}} e^{w(u,-i \bar{t})+i \bar{t} u} d t
$$

Hence,

$$
\begin{aligned}
& I_{5}^{\prime}-\frac{x^{\tilde{\beta}}}{\tilde{\beta} \log y} J_{5}^{\prime}= \\
& x^{\tilde{\beta}} Q(u) \cdot \frac{1}{2 \pi} \int_{-T_{b}}^{T_{b}} e^{w(u,-i \bar{t})+i \bar{t} u}\left(\frac{1}{B+i t}-\frac{1}{\tilde{\beta}}+O(R(y, t, q))\right) d t .
\end{aligned}
$$

By Lemmas 4 and 5 , it is

$$
\begin{aligned}
& \ll x^{\tilde{\beta}} Q(u) \int_{0}^{1 / \log y} e^{-c_{6} u \bar{t}^{2}}(\xi(u) / \log y+t) d t \\
& +x^{\tilde{\beta}} Q(u) \int_{1 / \log y}^{T_{b}} e^{-c_{9} u / \log ^{2} u}\left(\frac{\xi(u)}{\log y}+t\right) d t \ll \frac{x^{\tilde{\beta}} Q(u)}{\sqrt{u} \log y} \cdot \frac{\xi(u)}{\log y} .
\end{aligned}
$$

Combining these estimates, we obtain (5.12) for $j=5$ and hence the estimate (1.13) of Theorem 1 (ii), in the range considered.

The proof of Theorem 1 is completed.

\section{$\S 6$. Proofs of Theorem 2 and Corollary 1}

\section{Proof of Theorem 2}

We first consider the case:

$$
\exp \left\{(\log x)^{2 / 5+\varepsilon}\right\}<y \leq x^{1 / 2} .
$$

We have

$$
\Phi(x, y ; a, q)=\frac{1}{\varphi(q)}\left\{\Phi_{q}(x, y)+\sum_{\chi \neq \chi_{0}} \bar{\chi}(a) \sum_{n \in S(x, y)} \chi(n)\right\} .
$$

From this, Theorem 1 (i) and (ii), (1.15) follows. (We note that for $P(q) \leq y$ we have $\Phi_{q}(x, y)=\Phi(x, y)$.) 
We now consider the case: $x^{1 / 2}<y \leq x / q$. By the prime number theorem of arithmetic progressions, we have for $q$ satisfying (1.18)

$$
\begin{aligned}
(6.3) \Phi(x, y ; a, q) & =\sum_{y<p \leq x} 1 \\
& =\frac{l i x-l i y}{\varphi(q)}-\frac{\tilde{\chi}(a)}{\varphi(q)} \int_{y}^{x} \frac{v^{\tilde{\beta}-1}}{\log v} d v+O\left(x e^{-C^{\prime} \sqrt{\log x}}\right) .
\end{aligned}
$$

We remark that for $1<u \leq 2$ we have $\rho^{\prime}(u)=-1 / u$. Also, $y^{\tilde{\beta}} \ll x / \sqrt{\log x}$, since $y \leq x / q \leq x / q_{1} \ll x / \sqrt{\log x}$, by (1.21). From this, (1.14) (where $c_{3}$ has been chosen sufficiently small) and the prime number theorem, (1.15) follows.

$$
\text { If }
$$

$$
3 / 2 \leq y \leq \exp \left\{(\log x)^{2 / 5+\varepsilon}\right\}
$$

we have

$$
\rho(u)=e^{-u \log u(1+o(1))} \ll e^{-\sqrt{\log x}}, \quad \text { and } \quad \rho^{\prime}(u) \sim-\log u \cdot \rho(u),
$$

hence (1.15) follows from this and (1.7).

This completes the proof of Theorem 2.

\section{Proof of Corollary 1}

To deduce Corollary 1 from Theorem 2 for $x, y$ satisfying (6.1), it suffices to show

$$
x^{\tilde{\beta}} \ll x e^{-\sqrt{\log x}}
$$

holds uniformly in the range (1.17).

By Siegel's theorem, for any $\varepsilon>0$ there exists a positive number $c(\varepsilon)$ such that $\tilde{\beta} \leq 1-c(\varepsilon) q^{-\varepsilon}$. Put $\varepsilon=1 /(2 A+1),(6.5)$ follows, hence the proof of Corollary 1 is completed in the range considered.

If $x^{1 / 2}<y \leq x / q$, the estimate (1.16) follows from the prime number theorem of arithmetic progressions (the second term in (1.19) have been deleted) and the prime number theorem.

If $x, y$ satisfying (6.4), the estimate (1.16) follows immediate from (1.7). This completes the proof of Corollary 1.

Acknowledgements. The author expresses his thanks to the referee for his comments and suggestions. 


\section{REFERENCES}

[1] N. G. de Bruijn, On the number of uncancelled elements in the sieve of Eratosthenes, Nederl. Akad. Wetensch. Proc. Ser. A, 53 (1950), 803-812.

$[2] \longrightarrow$, The asymptotic behavior of a function occurring in the theory of primes, J. Indian Math. Soc. (N. S.), 15 (1951), 25-32.

[3] - On the number of positive integers $\leq x$ and free of prime factors $>y$, Nederl. Akad. Wetensch. Proc. Ser. A, 54 (1951), 50-60.

[4] A. A. Buchstab, Asymptotic estimates of a general number-theoretic function (Russian), Mat. Sb., 2(44) (1937), 1239-1246.

[5] H. Davenport, Multiplicative Number Theory (2nd Edn.), 74, GTM, SpringerVerlag, New York, 1980.

[6] E. Fouvry and G. Tenenbaum, Entiers sans grand facteur premier en progressions arithmétiques, Proc. London Math. Soc., (3)63 (1991), 449-494.

[7] J. Friedlander and A. Granville, Limitations to the equi-distribution of prime I, Ann. Math., 129 (1989), 363-382.

[8] J. Friedlander, A. Granville, A. Hildebrand and H. Maier, Oscillation theorems for primes in arithmetic progressions and for sifting functions, J. Amer. Math. Soc., 4 (1991), 25-86.

[9] H. Halberstam and H. -E. Richert, Sieve Methods, Academic Press, London, New York, 1974.

[10] A. Hildebrand, The asymptotic behavior of the solutions of a class of differential-difference equation, J. London Math. Soc., 42 (1990), 11-31.

[11] A. Hildebrand and H. Maier, Irregularities in the distribution of primes in short intervals, J. Reine Angew. Math., 397 (1989), 162-193.

[12] A. Hildebrand and G. Tenenbaum, On integers free of large prime factors, Trans. Amer. Math. Soc., 296 (1986), 265-290.

[13] H. Iwaniec, Rosser's sieve, Acta Arith., 36 (1980), 171-202.

[14] H. Maier, Primes in short intervals, Michigan Math. J., 32 (1985), 221-225.

[15] K. K. Norton, 'Numbers with small prime factors and the least $k$ th power non residue, 106 (1971), Mem. Amer. Math. Soc.

[16] C. D. Pan and C. B. Pan, Elements of Analytic Number Theory (Chinese ), Scientia Press, Beijing, 1991.

[17] K. Prachar, Primzahlverteilung, Springer-Verlag, Berlin, 1957.

[18] E. Saias, Sur le nombre des entiers sans grand facteur premier, J. Number Theory, 32 (1989), 78-99.

[19] G. Tenenbaum, Introduction to analytic and probabilistic number theory, Cambridge studies in advanced mathematics, No. 46, Cambridge University Press, 1995.

[20] E. C. Titchmarsh, The theory of the Riemann-Zeta function ( $2^{\text {nd }}$ edition, revised by D. R. Heath-Brown, Oxford, 1986.

[21] A. I. Vinogradov, On numbers with small prime divisors, (Russian), Dokl. Akad, Nauk SSSR (N. S.), 109 (1956), 683-686.

[22] D. Wolke, Über die mittlere verteilung der werte zahlenthloretischer funktionen, Math. Ann., 204 (1973), 145-153. 
[23] T. Z. Xuan, On the asymptotic behavior of the Dickman-de Bruijn function, Math. Ann., 297 (1993), 519-533.

[24] On the asymptotic estimates of sifting function, Quart. J. Math. Oxford (2), 49 (1998), 237-258.

Department of Mathematics

Beijing Normal University

Beijing 100875

People's Republic of China 